\title{
NOVA OCORRÊNCIA DE MEGAFAUNA PLEISTOCÊNICA EM MATO GROSSO DO SUL
}

\author{
Thaís Rabito PANSANI
}

Alessandro Marques de OLIVEIRA

Mírian Liza Alves Forancelli PACHECO

\begin{abstract}
RESUMO
O estado do Mato Grosso do Sul, Brasil, tem revelado um importante potencial paleontológico dos depósitos quaternários, em especial devido à riqueza de fósseis encontrados nas grutas e rios da região da Serra da Bodoquena. Este trabalho apresenta novos dados taxonômicos de espécimes coletados do rio Miranda (Miranda, MS), uma nova localidade fossilífera da megafauna pleistocênica da região. Dentre os materiais identificados, estão restos fósseis de Megatheriidae, Mylodontidae, Glyptodontinae, Pampatheriidae e Toxodontidae. Ressalta-se a ocorrência, até agora inédita, do pampaterídeo Holmesina, que preenche uma lacuna paleobiogeográfica na América do Sul.
\end{abstract}

Palavras-chave: Megafauna; Serra da Bodoquena; Pleistoceno; Mato Grosso do Sul.

\section{ABSTRACT}

\section{NEW OCCURRENCE OF PLEISTOCENE MEGAFAUNA IN MATO GROSSO} DO SUL. The state of Mato Grosso do Sul, Brazil, has revealed an important paleontological potential of Quaternary deposits, especially because of the fossil richness in caves and rivers of the Serra da Bodoquena region. This study presents new taxonomic data of specimens collected from a new fossiliferous locality of the Pleistocene megafauna in this region, the Miranda River (Miranda, MS). Among the material that has been identified are fossil remains of Megatheriidae, Mylodontidae, Glyptodontinae, Pampatheriidae and Toxodontidae. It is important to mention the novel occurrence of the Pampatheriidae Holmesina, which fills a paleobiogeographic gap in South America.

Keywords: Megafauna; Serra da Bodoquena; Pleistocene; Mato Grosso do Sul.

\section{INTRODUÇÃO}

O potencial fossilífero de Mato Grosso do Sul (MS) coloca este estado em posição de destaque no cenário paleontológico nacional e internacional. Suas paisagens abarcam afloramentos com um dos mais antigos registros fósseis de animais no mundo em Corumbá e Ladário (BECKER-KERBER et al. 2013, PACHECO et al. 2015), os invertebrados marinhos do Devoniano da Bacia do Paraná, em Rio Verde (BECKER-KERBER et al. 2017), além das pegadas de dinossauros no município de Nioaque (SCHEFFLER et al. 2010). Até o momento, poucos estudos sobre fósseis da megafauna pleistocênica foram feitos neste estado (OLIVEIRA et al. 2017).

Estudos taxonômicos e tafonômicos sobre fósseis de vertebrados encontrados nas cavernas da região da Serra da Bodoquena contribuíram para o conhecimento sobre a riqueza taxonômica e os processos de deposição de restos esqueléticos nestes locais durante o Pleistoceno e Holoceno (SALLES et al. 2006, OLIVEIRA 2013). PERINI et al. (2009) identificaram vários grupos de mamíferos pleistocênicos e holocênicos, como Felidae (Smilodon populator), Canidae (Chrysocyon brachyurus, Protocyon troglodytes) e Mustelidae (Pteronura brasiliensis), procedentes das cavernas submersas Japonês e Nascente do Formoso. Este estudo possibilitou uma primeira inferência paleoambiental, na qual, segundo os mesmos autores, a região da Serra da Bodoquena seria caracterizada por vegetação do tipo savana com áreas inundadas, semelhante ao Pantanal, durante a passagem Pleistoceno/Holoceno. 
OLIVEIRA (2013) identificou outros grupos de mamíferos de grande porte (e.g. Mylodontidae, Megatheriidae, Glyptodontidae, Ursidae) e um fêmur humano (Homo sapiens), que podem contribuir para a discussão sobre a coexistência das populações humanas pré-históricas com a megafauna. As ocorrências de um ursídeo, um provável Glyptotherium, e uma preguiça terrícola, provável Scelidodon tarijensis, podem fornecer novas interpretações paleobiogeográficas e paleoambientais, caso suas identificações sejam confirmadas.

Em 2014, novos fósseis da megafauna pleistocênica foram encontrados na Serra da Bodoquena, provenientes do leito do rio Miranda. Os materiais foram coletados durante o processo de dragagem dos sedimentos do rio e doados à Universidade Federal de São Carlos (Campus Sorocaba). Devido à escassez de estudos sobre fósseis de Mato Grosso do Sul nas temáticas supracitadas, faz-se necessário um estudo taxonômico sobre os mesmos. Portanto, o objetivo deste trabalho foi identificar os restos da paleofauna encontrada no leito do rio Miranda, uma nova localidade fossilífera da megafauna em Mato Grosso do Sul.

\section{CONTEXTO GEOLÓGICO}

O estado de Mato Grosso do Sul, localizado na região Centro-Oeste do país, compreende três entidades geotectônicas: o Cráton Amazônico, a Província Tocantins e as bacias sedimentares fanerozoicas (SCHEFFLER et al. 2010), com destaque para a bacia quaternária do Pantanal. Na Formação Pantanal, na bacia homônima, os registros fossilíferos mais abundantes são associados à megafauna pleistocênica (SCHEFFLER et al. 2010).

Sustentada por rochas calcárias do Grupo Corumbá, contornando o Cráton Amazônico (BOGGIANI \& COIMBRA 1998), a Serra da Bodoquena (Figura 1) possui cerca de $200 \mathrm{~km}$ de extensão e largura que varia entre 10 a $70 \mathrm{~km}$ (BOGGIANI et al. 2008). Encontra-se na bacia hidrográfica do rio Paraguai, abrangendo as sub-bacias dos rios Miranda e Aquidauna. Apresenta-se íngreme a oeste, em direção ao Pantanal, e caimento leve para leste, na transição com a planície de inundação do rio Miranda (BOGGIANI \& CLEMENTE 1999).

A presença de rochas carbonáticas do substrato rochoso influencia a paisagem da Serra da Bodoquena, que, por serem solúveis, favorecem a formação de cavernas, dolinas e outras feições de relevo cárstico (OLIVEIRA 2013). Seu carste é caracterizado por salões e condutos submersos, com nascentes e rios com tufas calcárias em crescimento, e diversas cachoeiras e represas naturais (BOGGIANI et al. 2011).
A Depressão do Rio Miranda (ALMEIDA 1965) é um compartimento geomorfológico que compõe a Serra da Bodoquena, constituindo uma extensa área rebaixada (cota 100-350 m), limitada a leste pelo Planalto de Maracaju (ALVARENGA et al. 1982). O carste nesta área é composto por morros calcários residuais (cones cársticos) e dolinas.

Os fósseis estudados neste trabalho provêm do leito do rio Miranda, numa localidade próxima à entrada do município de Miranda, onde está localizado o empreendimento Areeiro Silva (rodovia MS-339), nas coordenadas geográficas $20^{\circ} 14^{\prime} 27.30^{\prime \prime} \mathrm{S} / 56^{\circ} 23^{\prime} 59.30^{\prime \prime} \mathrm{O}$ e altitude de $115 \mathrm{~m}$.


FIGURA 1 - Ponto de coleta das amostras fossilíferas no rio Miranda, região da Serra da Bodoquena, Mato Grosso do Sul, Brasil.

\section{MATERIAIS E MÉTODOS}

Os fósseis foram encontrados por moradores da região em meio aos sedimentos retirados do leito do rio Miranda, durante o processo de dra- 
gagem do rio, e posteriormente foram repassados aos autores durante campanhas de campo em 2014. Todos os fósseis aqui estudados encontram-se depositados na Coleção Arqueológica e Paleontológica (sigla CAP) da Universidade Federal de São Carlos, Campus Sorocaba, SP, sob os números de tombo: CAP/1B-351, CAP/1B-395, CAP/1B-345, CAP/1B-342, CAP/1B-344A， CAP/1B-344B, CAP/1B-338A, CAP/1B-338B e CAP/1B-355.

As amostras foram triadas por morfotipos e identificadas até a categoria taxonômica mais específica possível, observando-se aspectos paleoautoecológicos, como os estágios ontogenéticos, por meio da evidência de linhas epifisárias e labeamentos. Para identificação taxonômica dos espécimes seguiu-se a literatura especializada, como os trabalhos de PAULA COUTO (1979), DE IULIIS (1996), BARGO \& VIZCAÍNO (2008), OLIVEIRA \& PEREIRA (2009), PITANA et al. (2013), GÓIS et al. (2015).

\section{SISTEMÁTICA PALEONTOLÓGICA}

Superordem XENARTHRA Cope, 1889

Ordem TARDIGRADA Latham \& Davies, 1795

Superfamília MEGATHERIOIDEA Gray, 1821

Família MEGATHERIIDAE Owen, 1843

Subfamília MEGATHERIINAE Gill, 1872

Gênero Eremotherium Spillmann, 1948

Espécie Eremotherium laurillardi (Lund, 1842)

Cartelle \& Bohórquez, 1982

Material: Calcâneo CAP/1B-351.

Descrição e comentários: O material (Figura 2) trata-se de um calcâneo de Eremotherium laurillardi. Apresenta estruturas visíveis para identificação do megatério, tais como seu aspecto alongado e relativamente estreito posteriormente, característico de
E.laurillardi (DE IULIIS 1996). É possível observar no espécime CAP/1B-351 a região onde se encontram as facetas sustentacular (articular interna) e ectal (articular externa), ambas de superfície lisa, divididas por uma porção relativamente mais profunda, o sulcus talis, de formato relativamente oval, e que se dispõe entre as facetas de forma dorsal. A partir dessa região, o osso estende-se para a região de tuber calcis, de aspecto mais rugoso (PAULA COUTO 1979). A textura apresenta-se predominantemente rugosa no resto do material. O completo desaparecimento de linhas epifisárias e a presença de labeamentos nas extremidades desse osso corroboram tratar-se de um indivíduo senil. Esta família possui ampla distribuição no território brasileiro, sendo encontrada em quase todos os estados do país: Rio de Janeiro (PRICE \& CAMPOS 1970), São Paulo (PAULA COUTO 1973, GHILARDI 2011, GHILARDI et al. 2011), Minas Gerais (MARTINELLI et al. 2012), Espírito Santo (PAULA COUTO 1978, GHILARDI 2011), Rio Grande do Norte (OLIVEIRA \& DAMASCENO 1987), Acre (SIMPSON \& PAULA COUTO 1981), Piauí (FAURE et al. 1999), Rondônia (NASCIMENTO 2008, GHILARDI 2011), Mato Grosso do Sul (SCHEFFLER et al. 2010), Goiás (PAULO \& BERTINI 2013), Paraná (DA SILVA 2008), Rio Grande do Sul (CARTELLE et al. 2015), Alagoas (DIAS NETO et al. 2008) Pernambuco (SILVA et al. 2010), Sergipe (DANTAS et al. 2011), Ceará (ARAÚJO JÚNIOR et al. 2012), Bahia (DANTAS et al. 2013). Para o restante da América do Sul, há registros desta família na Colômbia (DE PORTA 1961), Equador e Venezuela (CARTELLE 2000), Bolívia (SAINT-ANDRE \& DE IULIIS 2001), Peru (PUJOS \& SALAS 2004), Argentina (FERRERO et al. 2007) e Chile (ROJAS et al. 2012).
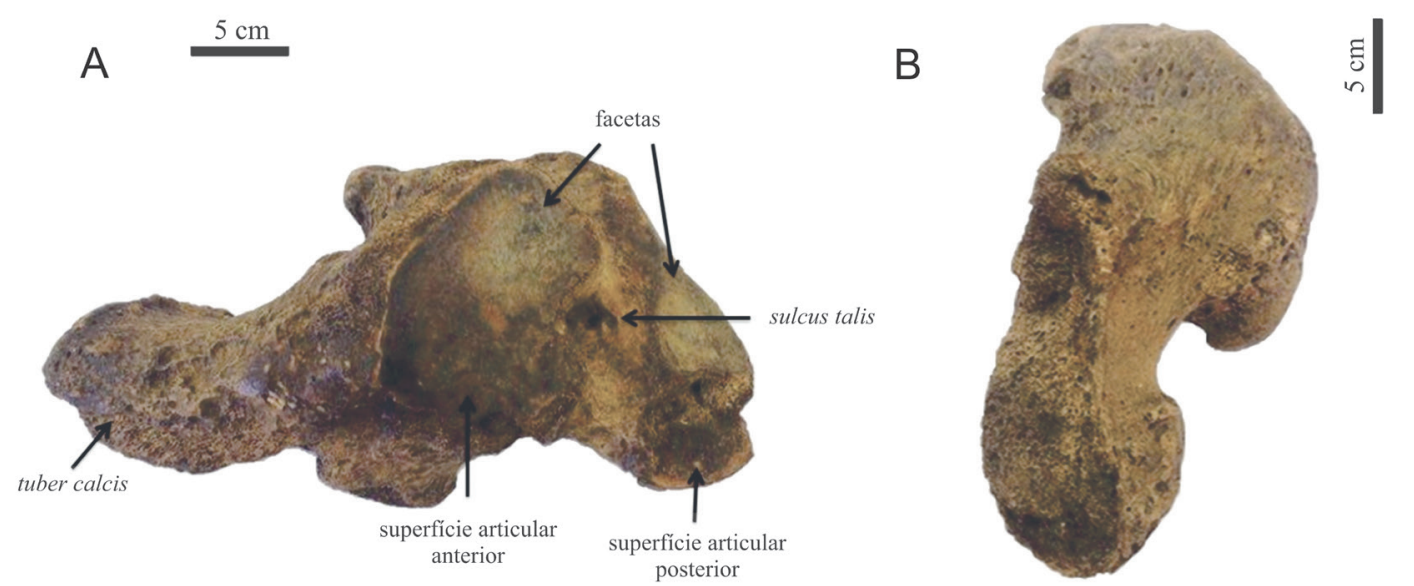

FIGURA 2 - Calcâneo de Eremotherium laurillardi CAP/1B-351 com estruturas descritas. A) vista dorsal e B) vista ventral. 
Família MYLODONTIDAE Ameghino, 1889 Subfamília MYLODONTINAE Ameghino, 1889 Gênero Glossotherium Owen, 1840

Material: Fragmento de molar CAP/1B - 395. Descrição e comentários: Dentre os representantes da família Mylodontidae, figura um fragmento desgastado de primeiro molar (M1) de Glossotherium (Figura 3), característico pelo seu formato subtriangular em vista oclusal, camada externa do seu cemento fina, superfícies de oclusão dos molariformes côncavas, camada externa da dentina rija, tornando-a mais afia$\mathrm{da}$, e superfícies lingual e labial amplas (BARGO \& VIZCAÍNO 2008, PITANA et al. 2013). Mylodontidae tem registro nos estados brasileiros: Acre (SIMPSON \& PAULA COUTO 1981), São Paulo (CARVALHO 1952), Minas Gerais, Rio Grande do Sul (PAULA COUTO 1979), Mato Grosso do Sul (SALLES et al. 2006), Bahia, Piauí, Sergipe, Alagoas, Ceará e Paraíba (GHILARDI 2011). Na América do Sul: Uruguai, Chile, Equador (PAULA COUTO 1979), Argentina (FERRERO et al. 2007), Venezuela (RINCÓN et al. 2015).

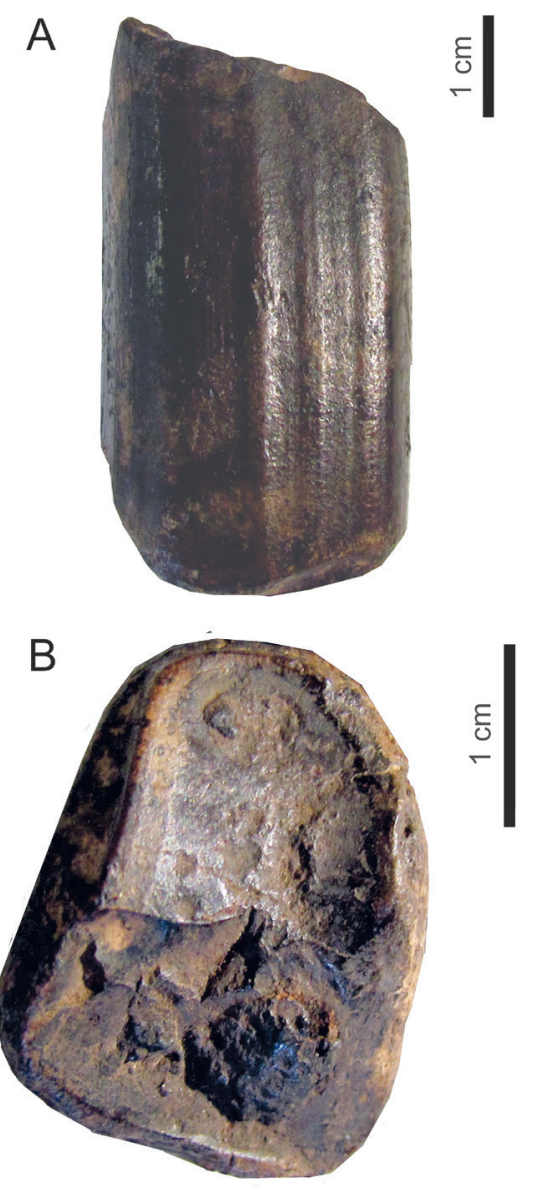

FIGURA 3 - Fragmento de dente de Glossotherium, CAP/1B-395. A) vista lingual e B) vista oclusal.
Superordem XENARTHRA Cope, 1889

Ordem CINGULATA Illiger, 1811

Família GLYPTODONTIDAE Burmeister, 1879

Subfamília GLYPTODONTINAE Trouessart, 1898

Material: Quatro osteodermos dorsais, CAP/1B-345, CAP/1B-342, CAP/1B-344A e CAP/1B-344B.

Descrição e comentários: Os cingulados abrangem os tatus e gliptodontes. O grupo possui carapaça de placas ósseas que cobre o dorso e flancos e separa o topo da cabeça e a cauda. As placas dérmicas podem ser tetra, penta ou hexagonais, cujas ornamentações podem variar de gênero e/ ou espécie (PAULA COUTO 1979). As amostras estudadas neste trabalho possuem um clássico padrão que se assemelha tanto a Glyptodon quanto a Glyptotherium: em formato de roseta, com variação de sete a treze figuras periféricas, superfície externa uniformemente pontuada por pequenos orifícios e sulco principal delimitado por forames pilosos (Figura 4). O diâmetro dos osteodermos varia entre 4 e $7 \mathrm{~mm}$. Em algumas das amostras (CAP/1B-345, CAP/1B-342) é possível visualizar a ornamentação bem definida: hexagonal com sulco circular central envolto por figuras periféricas menores, que variam em número de 5 a 8 , além dos folículos pilosos entre os sulcos radiais, presentes em número entre 4 e 7 unidades. Em outras (CAP/1B-344A e CAP/1B344B), devido a um maior grau de abrasão, essas estruturas são menos visíveis. CAP/1B-345 apresenta uma porção central circular mais elevada que os periféricos, porém, o padrão típico hexa ou pentagonal ainda pode ser identificado, assim como em outras amostras de osteodermos, se observadas minuciosamente. OLIVEIRA et al. (2010) propõem que a maioria dos espécimes identificados no Brasil como Glyptodon trata-se, na realidade, do gênero norte-americano Glyptotherium, que participou do Grande Intercâmbio Biótico Americano (GIBA). A utilização de apenas osteodermos isolados para identificação é um dos principais geradores de dúvidas e incertezas na taxonomia destes gêneros (ZURITA et al. 2011), portanto, é importante realizar diagnoses a partir de métodos osteológicos ou a partir de demais elementos fósseis (por exemplo, crânio e dentes). Desse modo, embora os materiais aqui analisados possuam características semelhantes à Glyptodon, mas não há fragmentos fósseis adicionais para sustentar esta identificação, deixamos o gênero do material em aberto, apenas a nível de subfamília Glyptodontinae. 
Glyptodontinae apresenta distribuição em grande parte do território brasileiro, nos estados de: Mato Grosso do Sul (SALLES et al. 2006), Rio Grande do Sul, São Paulo, Minas Gerais, Mato Grosso, Rondônia, Acre, Piauí, Bahia, Rio Grande do Norte, Sergipe, Pernambuco (GHILARDI
2011), Paraíba (BERGQVIST 1989) e Tocantins (AVILLA et al. 2012). Na América do Sul, também foram identificados na Argentina, Bolívia, Chile, Paraguai (MIÑO-BIOLINI et al. 2005), Uruguai, Colômbia, Venezuela e Peru (ZURITA et al. 2012).
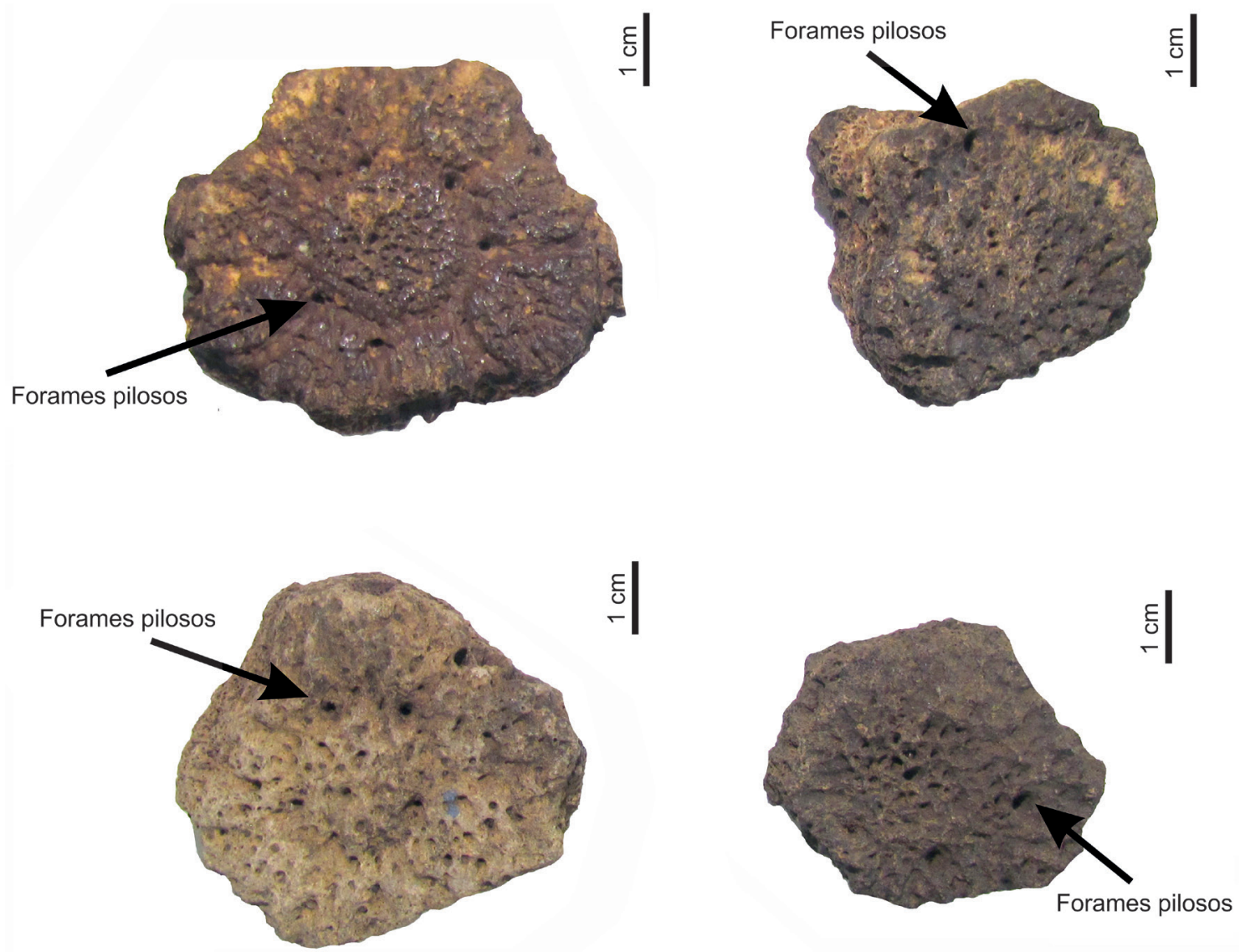

FIGURA 4 - Osteodermos de Glyptodontinae. A: CAP/1B-345, B: CAP/1B-342,C: CAP/1B-344A e D: CAP/1B-344B. Vista dorsal.

Ordem CINGULATA Illiger, 1811 Família PAMPATHERIIDAE Paula Couto, 1954

Gênero Holmesina Simpson, 1930

Material: Dois osteodermos dorsais, CAP/1B-338A e CAP/1B-338B.

Descrição e comentários: A família Pampatheriidae caracteriza-se por seus osteodermos diferenciados (GÓIS et al. 2015). Nota-se que suas placas osteodérmicas são alongadas, quadrangulares, podendo ser penta ou hexagonais, de face externa rugosa e ornamentações com fossetas (PAULA COUTO 1979). Foram observados osteodermos típicos de pampaterídeos, com ornamentações diferenciadas, margens lateral e anterior mais amplas e robustas, com depressões longitudinais e elevação por todo o perímetro central (GÓIS et al. 2015). As placas dérmicas identificadas neste trabalho (Figura 5) assemelham-se àquelas descritas por OLIVEIRA \& PEREIRA (2009), tanto em tamanho quanto em forma, caracterizando assim o material como Holmesina. Apresenta formato retângulo quadrangular, com 
superfície externa rugosa e marcada por pequenas perfurações, com diâmetro que varia entre $43 \mathrm{~mm}$ e $57 \mathrm{~mm}$ e espessura entre $10 \mathrm{~mm}$ e $16 \mathrm{~mm}$. As margens são relativamente amplas. Em adição, apresentam as elevações central e lateral bem definidas. Essa família possui representantes datados desde o Mioceno Médio até o Holoceno inicial na América do Sul e, a partir do Grande Intercâmbio Biótico Americano, migraram para a América do Norte entre o Plioceno tardio e o Pleistoceno tardio, quando diversificaram em pelo menos cinco gêneros, incluindo Holmesina (GÓIS et al. 2012).



A maior diversificação de cingulatos pleistocênicos em território brasileiro ocorreu no estado do Rio Grande do Sul (OLIVEIRA et al. 2009). No Brasil, remanescentes de pampaterídeos são documentados nos estados de Minas Gerais, Bahia, Ceará, Piauí, Rondônia, Mato Grosso, Santa Catarina, Rio Grande do Sul (GÓIS et al. 2012), Tocantins (AVILLA et al. 2012) e no restante da América do Sul, no Uruguai, Paraguai, Bolívia, Argentina, Colômbia, Venezuela, Equador (GÓIS et al. 2012) e Peru (PUJOS \& SALAS 2004, GÓIS et al. 2012).

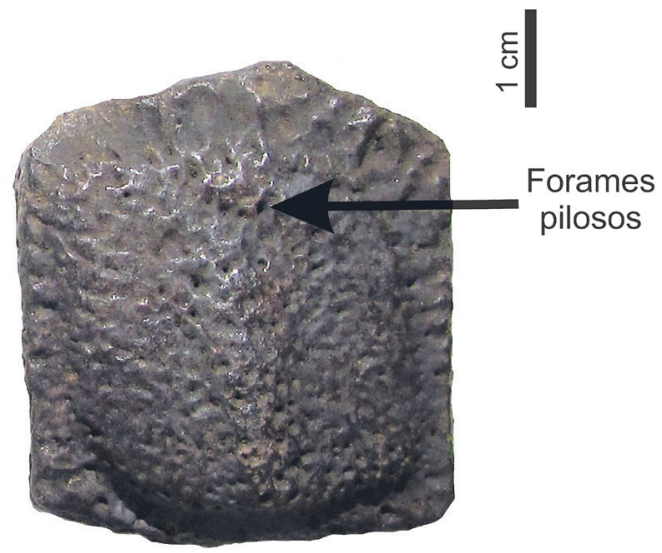

FIGURA 5 - Osteodermos de Holmesina, CAP/1B-340 e CAP/1B-338B. Vista dorsal.

Ordem NOTOUNGULATA Roth, 1903

Subordem TOXODONTIA Owen, 1853

Família TOXODONTIDAE Owen, 1845

Subfamília TOXODONTINAE Owen, 1845

Gênero Toxodon Owen, 1840

Material: Fragmento de molar superior CAP/1B-355. Descrição e comentários: Nota-se uma concavidade externa no fragmento do molar analisado (Figura 6). O fragmento possui $20,5 \mathrm{~cm}$ de comprimento. Há nítida preservação de partes da coloração do esmalte (Figura 6A), com disposição de camadas bem definidas verticalmente. $\mathrm{O}$ esmalte de seus dentes dispõe-se em faixas verticais, intercaladas com outras sem esmalte, especialmente na camada interna dos dentes. Conforme a descrição de PAULA COUTO (1979), identificou-se um sulco bifurcado. Este separa o protolofo e o metalofo, formando um curto "Y" em vista oclusal (Figura 6C). Ressalta-se que na vista oclusal é possível observar que algumas partes anatômicas encontram-se fragmentadas (protolofo, metalofo, parastilo, vale lingual). Porém, o ectolofo encontra-se intacto (Figura $6 \mathrm{C}$ ). Embora as demais partes estejam fragmentadas, é possível identificá-lo em função desta última característica como um dente molar superior de Toxodon. Conhecidos desde o Oligoceno Inferior até o final do Pleistoceno (PAULA COUTO 1979), os toxodontes são bem distribuídos no Brasil, com registros nos estados de São Paulo (GHILARDI et al. 2011), Minas Gerais, Rio Grande do Sul, Paraná, Rondônia, Mato Grosso, Bahia, Sergipe, Rio Grande do Norte, Pernambuco, Paraíba (MENDONÇA 2007, GHILARDI 2011), Rio de Janeiro (AVILLA et al. 2008), Mato Grosso do Sul (SALLES et al. 2006, OLIVEIRA 2013), Acre, Ceará, Piauí, Alagoas e Santa Catarina (GHILARDI 2011). Em outras partes da América do Sul, ocorrem na Venezuela (BOND et al. 2006), Argentina, Bolívia, Paraguai e Uruguai (PAULA COUTO 1979). 
A

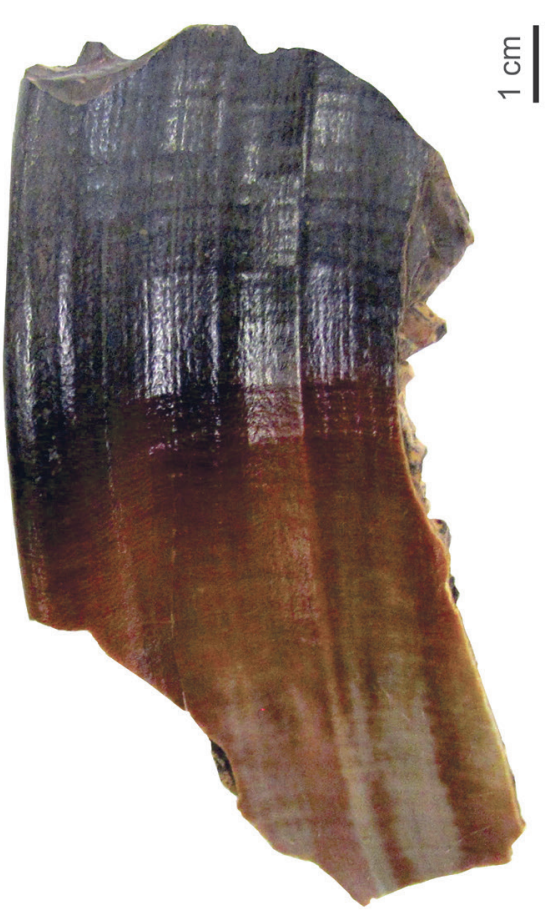

B

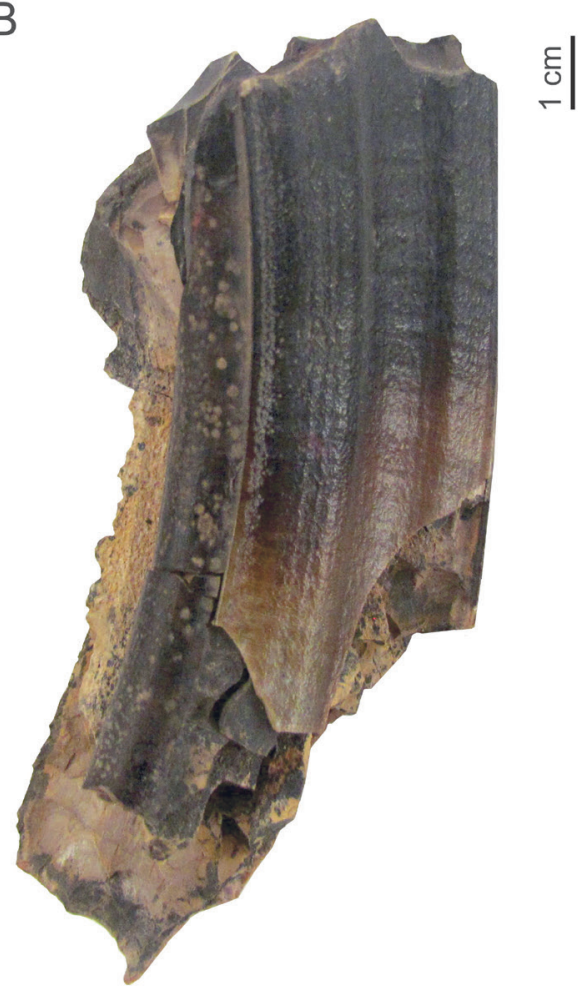

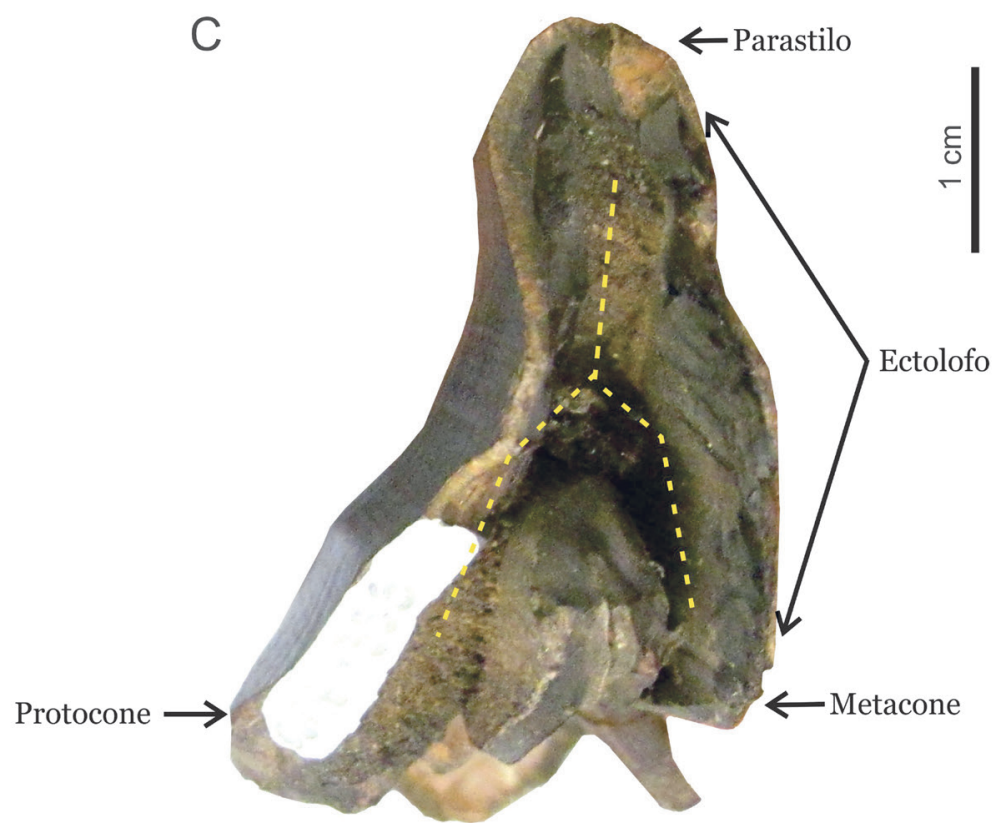

FIGURA 6 - Fragmento de molar de Toxodon, CAP/1B-355. A) vista labial, B) vista lingual, C) vista oclusal. 


\section{DISCUSS ÃO}

Estudos morfológicos do aparato mandibular e análises de isótopos estáveis mostram que Glyptodon e Glyptotherium possuiam hábitos pastadores de gramíneas, com dieta composta por plantas $\mathrm{C}_{3}$ e $\mathrm{C}_{4}$, predominando plantas $\mathrm{C}_{4}$ (FARIÑA \& VIZCAÍNO 2001, PÉREZ-CRESPO et al. 2012, DANTAS et al. 2013). Consequentemente, uma interpretação plausível seria considerar que estas espécies teriam habitado áreas de vegetação aberta (PÉREZ-CRESPO et al. 2012). Eremotherium laurilardi apresentava uma dieta mista, englobando plantas $\mathrm{C}_{4}$ (herbáceas, gramíneas) e $\mathrm{C}_{3}$ (frutos e folhas), assim como Toxodon (MACFADDEN 2005, DANTAS et al. 2013, FRANÇA et al. 2014, LOBO et al. 2015). Por esta razão, alguns autores propuseram a possibilidade destes táxons terem habitado tanto áreas abertas como bordas de florestas (MACFADDEN 2005, FRANÇA et al. 2014 DANTAS \& COZZUOL 2016). Holmesina (Pampatheriidae) e Glossotherium (Mylodontidae) são interpretados como formas pastadoras de gramíneas, evidenciado por características morfológicas, análises de isótopos de carbono e padrões de desgaste dentário tipicamente encontrados em animais escavadores, propiciados pela ingestão de partículas de poeira e solo (VIZCAÍNO et al. 1998, DE IULIIS et al. 2000, BARGO et al. 2006).

SALLES et al. (2006) sugeriram um cenário hipotético para Serra da Bodoquena durante o Pleistoceno final baseado na presença de fósseis de mamíferos adaptados para viver em savanas e também de táxons associados a ambientes florestais. Sendo assim, os autores consideraram a possibilidade da região ter sido composta por maiores áreas de vegetação aberta, subsistindo fragmentos de florestas. Os dados disponíveis na literatura para a alimentação preferencial dos táxons aqui identificados são sugestivos de uma interpretação que considere a presença de ambientes mais secos na Serra da Bodoquena durante o Pleistoceno final. Portanto, análises de isótopos estáveis nos materiais aqui estudados deverão auxiliar em interpretações paleoambientais locais.

Em um trabalho de revisão taxonômica, OLIVEIRA et al. (2010) restringiram a distribuição de Glyptodon em território brasileiro ao estado do Rio Grande do Sul, enquanto na região intertropical as ocorrências seriam atribuídas a Glyptotherium. ZURITA et al. (2012) levantaram a hipótese de ambos os gêneros terem compartilhado a mesma distribuição geográfica nas regiões centro-norte e leste da América do Sul.

Toxodon e Eremotherium laurillardi são mamíferos pleistocênicos que possuem uma das mais amplas faixas de distribuição na América do Sul (PAULA COUTO 1979, CARTELLE \& DE IULIIS 1995, OLIVEIRA et al. 2009). O gênero Glossotherium têm o maior número de ocorrências na região Pampeana (Argentina). Todos os três táxons já haviam sido registrados na Serra da Bodoquena (SALLES et al. 2006, SCHEFFLER et al. 2010, OLIVEIRA et al. 2017), sendo reforçada neste estudo a presença dos mesmos no estado de Mato Grosso do Sul.

Até o momento, não havia registro para a ocorrência de Holmesina no estado do Mato Grosso do Sul. Este novo dado preenche uma lacuna paleobiogeográfica existente entre a Argentina e o norte do Brasil para Pampatheriidae (especialmente Holmesina), que abriga no Rio Grande do Sul a maior quantidade de representantes da família registrados no Brasil (OLIVEIRA et al. 2009).

Diversos autores consideram que a presença de Holmesina em território brasileiro poderia ser reflexo da invasão de formas norte-americanas durante o Grande Intercâmbio Biótico Americano (SCILLATO-YANÉ et al. 2005, CARLINI et al. 2008, OLIVEIRA et al. 2009): uma reintrodução no continente sulamericano após diferenciações ocorridas nos pampaterídeos na América do Norte, onde migraram inicialmente. Uma possibilidade bastante plausível seria a de que Holmesina teria alcançado regiões de baixas latitudes (neste caso, a Serra da Bodoquena) durante o Pleistoceno final, em busca de melhores condições climáticas, seguindo uma leva de migração que abarcou diversos mamíferos partindo de regiões mais frias para regiões mais quentes (PRADO et al. 1987, GÓIS et al. 2012).

Adicionalmente, a presença inédita de Holmesina, juntamente com os demais táxons previamente conhecidos para o Mato Grosso do Sul, em território fora da Região Intertropical Brasileira (RIB) proposta por CARTELLE (1999), pode levar à reconsideração dos limites da RIB. Conforme recentemente apontado por OLIVEIRA et al. (2017), uma reformulação da RIB deveria incluir o estado de Mato Grosso do Sul. Datações absolutas serão importantes para testar hipóteses sobre sincronia e simpatria dos táxons do Mato Grosso do Sul em comparação com táxons de mamíferos pleistocênicos de outras regiões da América do Sul. 


\section{CONSIDERAÇÕES FINAIS}

Diante do exposto, considera-se que a Serra da Bodoquena, bem como o estado de Mato Grosso do Sul, possui em suas grutas e rios um alto potencial fossilífero, porém pouco explorado, que agrega novas informações ao acervo paleontológico brasileiro. Dentre as amostras identificadas, estão presentes restos fósseis de Megatheriidae, Mylodontidae, Glyptodontidae, Pampatheriidae e Toxodontidae. Reveste-se de importância a ocorrência de um espécime de pampaterídeo (Holmesina), uma novidade para o estado do Mato Grosso do Sul.

Um estudo mais aprofundado sobre os fósseis dessa região irá ajudar a compor a síntese de conhecimento sobre a megafauna do Pleistoceno no Brasil. Isto pode vir a auxiliar na elucidação de algumas questões ainda em aberto, como a extinção da megafauna pleistocênica na América do Sul, bem como a compreensão dos fatores ecológicos da época que podem ter influenciado nos padrões de distribuição geográfica das espécies.

\section{AGRADECIMENTOS}

Agradecemos ao Patrick Schaldach do Departamento Nacional de Produção Mineral de Campo Grande pelas informações fornecidas, à Dra. Sandra Gabas do Laboratório de Geologia da Universidade Federal de Mato Grosso do Sul pelo contato feito entre nós e o DNPM para a realização deste trabalho, aos membros da banca de TCC da autora Thaís Pansani, Dr. Rafael Delcourt e Prof. Dr. George Mattox, pelas críticas e sugestões que resultaram na elaboração do presente estudo, e aos alunos de graduação Gabriel Barros e Melissa Gusukuma, pela ajuda na organização e no tombamento da coleção de fósseis do laboratório. Também agradecemos ao Sr. Manoel Messias A. Silva, proprietário do empreendimento Areeiro Silva, pelo apoio durante as coletas em Miranda. Agradecemos às correções, críticas e sugestões científicas dos relatores e corpo editorial da Revista do Instituto Geológico para a realização deste trabalho.

\section{REFERÊNCIAS BIBLIOGRÁFICAS}

ALMEIDA, F.F.M. 1965. Geologia da Serra da Bodoquena (Mato Grosso), Brasil. Rio de Janeiro: Divisão de Geologia e Mineralogia, DNPM, 96 p. (Boletim, 219).

ALVARENGA, S.M.; BRASIL, A.E.; DEL'ARCO, D.M. 1982. Geomorfologia. In: Projeto RA-
DAMBRASIL, Ministério das Minas e Energia, Brasil, Levantamento de Recursos Naturais, vol. 28, Folha SF. 21 Campo Grande, Rio de Janeiro, p. 125-184.

ARAÚJO JÚNIOR, H.I.; BISSARO JÚNIR, M.C.; SANTOS, T.; ALVES, R.; BERGQVIST, L.P. 2012. Tafonomia da megafauna pleistocênica brasileira: Fluvial transport index (FTI) em análises de representatividade óssea. Revista Brasileira de Paleontologia, 15(1): 95-104.

AVILlA, L.S.; DOMINATO, V.H.; PAIXÃO, M.M.; ALVES-LEITE, M.; SOUZA-CRUZ, F.; GIL, B.B.; PESSÔA, J.B.; LIMA, D.T.; MONTEIRO, M.R.; FERREIRA, M.A.; MOTHÉ, D.; JERONYMO, C.L.; ROSA, D.T.; COSTA, D.G.; VASCONSCELLOS, K.L.; DELOQUE, L.; WINCK, G.R. 2008. Primeiros mamíferos quaternários encontrados em cavidades subterrâneas naturais do Estado do Rio de Janeiro. In: SBP, SIMPÓSIO BRASILEIRO DE PALEONTOLOGIA DE VERTEBRADOS, 6, Ribeirão Preto, Anais, 33.

AVILLA, L.; SOIBELZON, E.; CASTRO, M.; OLIVEIRA, E.V.; ZURITA, A.E. 2012. Os Cingulados (Xenarthra: Mammalia) fósseis registrados nas cavernas do sudeste do estado do Tocantins: taxonomia, aspectos climáticos e ambientais no Pleistoceno Superior do norte do Brasil. Paleontologia em Destaque Boletim Informativo da Sociedade Brasileira de Paleontologia, 36: 39.

BARGO, M.S.; VIZCAINO, S.F. 2008. Paleobiology of Pleistocene ground sloths (Xenarthra, Tardigrada): biomechanics, morphogeometry and ecomorphology applied to the masticatory apparatus. Ameghiniana, 45(1): 175-196.

BARGO, M.S.; DE IULLIS, G.; VIZCAÍNO, S.F. 2006. Hypsodonty in Pleistocene ground sloths. Acta Palaeontolica Polonica, 51(1): 53-61.

BECKER-KERBER, B.B.; ROSA, A.L.Z.; GABAS, S.G.; LEME, J.M.; PACHECO, M.L.A.F. 2013. O registro fossilífero de metazoários ediacaranos na América do Sul e duas implicações nos estudos sobre origem e complexificação da vida animal. Geologia USP, Série Cientifica, 13: 51-64.

BECKER-KERBER, B.; OSÉS, G.L.; CURADO, J.F.; RIZZUTTO, M.A.; RUDNITZKI, I.D.; ROMERO, G.R.; ONARY-ALVES, 
S.Y.; BENINI， V.G.; GALANTE， D.; RODRIGUES, F.; BUCK, P.V.; RANGEL, E.C.; GHILARDI, R.P.; PACHECO, M.L.A.F. 2017. Geobiological and diagenetic insights from Malvinokaffric Devonian biota (Chapada group, Paraná basin, Brazil): paleobiological and paleoenvironmental implications. Palaios, 4: 238-249.

BERGQVIST, L.P. 1989. Os mamíferos Pleistocênicos do Estado da Paraíba, Brasil, depositados no Museu Nacional, Rio de Janeiro. Programa de Pós-graduação em Zoologia, Museu Nacional, Universidade Federal do Rio de Janeiro, Rio de Janeiro, Dissertação de Mestrado, $174 \mathrm{p}$.

BOGGIANI., P.C.; CLEMENTE, J. 1999. A questão do licenciamento ambiental de empreendimentos turísticos no Planalto da Bodoquena-Mato Grosso do Sul. Revista de Geografia - UFMS, 9: 24-32.

BOGGIANI, P.C.; COIMBRA, A.M. 1998. Significado paleogeográfico da Superfície Pedra Branca na evolução da Bacia Corumbá (Neoproterozóico IIII). In: SBG, CONGRESSO BRASILEIRO DE GEOLOGIA, 40, Belo Horizonte, Anais, p. 45.

BOGGIANI, P.C.; SALLUN FILHO, W.; KARMANN, I.; GESICKI, A.L.; PHILADELPHI, N.M.; PHILADELPHI, M. 2008. Gruta do Lago Azul, Bonito, MS - Onde a luz do sol se torna azul. Sítios Geológicos e Paleontológicos do Brasil. Disponível em http://sigep.cprm.gov.br/sitio107/sitio107. pdf. Acessado em 10 dez. 2016.

BOGGIANI, P.C.; TREVELIN, A.C.; SALLUN FILHO, W.; OLIVEIRA, E.C.; ALMEIDA, L.H.S. 2011. Turismo e conservação de tufas ativas da Serra da Bodoquena, Mato Grosso do Sul. Tourism and Karst Areas: 4(1): 55-63.

BOND, M.; MADDEN, R.H.; CARLINI, A.A. 2006. A new specimen of Toxodontidae (Notoungulata) from the Urumaco formation (Upper Miocene) of Venezuela. Journal of Systematic Palaeontology, 4(3): 285-291.

CARLINI, A.A.; ZURITA, A.E.; AGUILERA, O. M. 2008. North American Glyptodontines (Xenarthra, Mammalia) in the Upper Pleistocene of northern South America. Palaontologische Zeitschrift, Palaontologische Zeitschrift, 82(2): 125-138.
CARTELLE, C. 1999. Pleistocene mammals of the Cerrado and Caatinga of Brazil. In: J.F. Eisenberg \& K.H. Redford (ed.) Mammals of the Neotropics. The University of Chicago Press, p. 27-46.

CARTELLE, C. 2000. Preguiças terrícolas, essas desconhecidas. Ciência Hoje, 27(161): 18-25.

CARTELLE, C.; DE IULIIS, G. 1995. Eremotherium laurillardi: the Panamerican Late Pleistocene Megatheriid Sloth. Journal of Vertebrate Paleontology, 15(4): 830-841.

CARTELLE, C.; DE IULIIS, G.; PUJOS, F. 2015. Eremotherium laurillardi (Lund, 1842) (Xenarthra, Megatheriinae) is the only valid megatheriine sloth species in the Pleistocene of intertropical Brazil: a response to Faure et al. 2014. Comptes Rendus Palevol, 14(1): 15-23.

CARVALHO, A.M.V. 1952. Ocorrências de Lestodon trigonidens na mamalofauna de Álvares Machado. São Paulo: Faculdade de Filosofia Ciências e Letras, Universidade de São Paulo, p. 43-55 (Boletim 134, Geologia 4).

DA SILVA, D.D. 2008. Os Xenarthra pleistocênicos do estado do Paraná. Setor de Ciências Biológicas, Universidade Federal do Paraná, Curitiba, Monografia, $37 \mathrm{p}$.

DANTAS, M.A.T.; COZZUOL, M.A. 2016. The Brazilian Intertropical Fauna from 60 to about 10 ka B.P.: Taxonomy, Dating, Diet and Paleoenvironments. In: G. Gasparini, J. Rabassa, E.P. Tonni, C. Deschamps, C. (ed.) Marine isotope stage 3 in southern South America, 60 ka B.P.-30 ka B.P. Springer, p. 207-226.

DANTAS, M.A.T.; PORPINO, K.O.; BAUERMANN, S.G.; PRATA, A.P.N.; COZZUOL, M.A.; KINOSHITA, A.; BARBOSA, J.H.O.; BAFFA, O. 2011. Megafauna do Pleistoceno Superior de Sergipe, Brasil: registros taxonômicos e cronológicos. Revista Brasileira de Paleontologia, 14(3): 311-320.

DANTAS, M.A.T.; MISSAGIA, R.; DUTRA, R.P.; RAUGUST, T.; SILVA, L.A.; DELICIO, M.P.; RENÓ, R. 2013. Identificação taxonômica dos fósseis de mamíferos da Caverna Toca Fria e Jatobá, Iuiú, Bahia: inferências paleoecológicas e temporais. In: SBE, CONGRESSO BRASILEIRO DE ESPELEOLOGIA, 32, Barreiras, Anais, 433-338. 
DE IULIIS, G. 1996. A Systematic Review of the Megatheriinae (Mammalia: Xenarthra: Megatheriidae). National Library of Canada, Toronto, $781 \mathrm{p}$.

DE IULIIS, G.; BARGO, M.S.; VIZCAÍNO, S.F. 2000. Variation in skull morphology and mastication in the fossil giant armadillos Pampatherium spp. and allied genera (Mammalia: Xenarthra: Pampatheriidae), with comments on their systematics and distribution. Journal of Vertebrate Paleontology, 20(4): 743-754.

DE PORTA, J. 1961. Edentata Xenarthra del Pleistoceno de Colombia. Nota Preliminar. Bucamanga: Universidad Industrial de Santander, Publicaciones Cientificas, p. 5-32 (Boletin de Geologia, 6).

DIAS NETO, C.M.; BORN, P.A.; CHAHUD, A. 2008. Occurrence of extinct mammals of the Pleistocene in Lagoa da Pedra, Igaci City, Alagoas State. Revista UnG - Geociências, 7(1): 110-115.

FARIÑA, R. A.; VIZCAÍNO, S. F. 2001. Carved teeth and strange jaws: how glyptodonts masticated. Acta Palaeontologica Polonica, 46(2): 219-234.

FAURE, M.; GUERIN, C.; PARENTI, F. 1999. The Holocene megafauna from the Toca do Serrote do Artur (São Raimundo Nonato archaeological area, Piauí, Brazil). Comptes Rendus de l'Academie des Sciences Series IIA Earth and Planetary Science, 329(6): 443-448.

FERRERO, B.; BRANDONI, D.; NORIEGA, J.I.; CARLINI, A. 2007. Mamiferos de la formacion El Palmar (Pleistoceno tardio) de la provincia de Entre Rios, Argentina. Revista del Museo Argentino de Ciencias Naturales, 9(2): 109-117.

FRANÇA, L.M.; ASEVEDO, L.; DANTAS, M.A.T.; BOCCHIGLIERI, A.; AVILLA, L.S.; LOPES, R.P.; SILVA, J.L.L. 2014. Review of feeding ecology data of Late Pleistocene mammalian herbivores from South America and discussions on niche differentiation. Earth-Science Reviews, 140: $158-165$.

GHILARDI, A.M. 2011. Megafauna do Quaternário Tardio dos depósitos cársticos do Alto Verde do Ribeira, sudeste do Estado de São Paulo, Brasil. Centro de Ciências Biológicas e Saúde, Universidade Federal de São Carlos, São Carlos, Dissertação de Mestrado, 122 p.
GHILARDI, A.M.; FERNANDES, M.A.; BICHUETTE, M.E. 2011. Megafauna from the Late Pleistocene-Holocene deposits of the Upper Ribeira karst area, southeast Brazil. Quaternary International, 245(2): 369-378.

GÓIS, F.; SCILLATO-YANÉ, G.J.; CARLINI, A.A.; UBILlA, M. 2012. Uma nueva espécie de Holmesina Simpson (Xenarthra, Cingulata, Pampatheriidae) del pleistoceno de Rondônia, sudoeste de la Amazonia, Brasil. Revista Brasileira de Paleontologia, 15(2): 211-227.

GÓIS, F.; RUIZ, L.R.G.; SCILLATO-YANÉ, G.J.; SOIBELZON, E.; BEATTY, B.L. 2015. A Peculiar New Pampatheriidae (Mammalia: Xenarthra: Cingulata) from the Pleistocene of Argentina and Comments on Pampatheriidae Diversity. PLoS One, 10(6) e0128296.

LOBO, L.S.; SCHERER, C.S.; DANTAS, M.A.T. 2015. Megafauna do pleistoceno final de Matina, Bahia, Brasil: sistemática, cronologia e paleoecologia. Revista Brasileira de Paleontologia, 18(2): 325-338.

MACFADDEN, B.J. 2005. Diet and habitat of toxodont megaherbivores (Mammalia, Notoungulata) from the late Quaternary of South and Central America. Quaternary Research, 64: 113-124.

MARTINELLI, A.G.; FERRAZ, P.F.; CUNHA, G.C.; CUNHA, I.S.; CARVALHO, I. S.; RIBEIRO, L.C.B.; MACEDO NETO, F.; CAVELLANI, C.L.; TEIXEIRA, V.P.A.; FERRAZ, M.L.F. 2012. First record of Eremotherium laurillardi (Lund, 1842) (Mammalia, Xenarthra, Megatheriidae) in the Quaternary of Uberaba, Triângulo Mineiro (Minas Gerais State), Brazil. Journal of South American Earth Sciences, 37: 202-207.

MENDONÇA, R. 2007. Revisão dos toxodontes pleistocênicos brasileiros e considerações sobre Trigodonops lopesi (Roxo, 1921) (Notoungulata, Toxodontidae). Instituto de Biociências, Universidade de São Paulo, São Paulo, Dissertação de Mestrado, 104 p.

MIÑO-BIOLINI, A.R.; CANTEROS, O.A.; ALCARAZ, M.A.; LUTZ, A.I. 2005. Últimos hallazgos de mamíferos fósiles del Arroyo Toropí (Pleistoceno Tardío Holoceno Temprano) y revisión sistemática de los Toxodontidae cuaternarios de la provincia de Corrientes, Argentina. Universidad Nacional 
del Nordeste, Comunicaciones Científicas y Tecnológicas, Resumen B-009.

NASCIMENTO, E.R. 2008. Os Xenarthra Pilosa (Megatheriidae), Notoungulata (Toxodontidae) e Proboscidea (Gomphotheriidae) da formação Rio Madeira, Pleistoceno Superior, Estado de Rondônia, Brasil. Instituto de Geociências, Universidade Federal do Rio Grande do Sul, Porto Alegre, Dissertação de Mestrado, 113 p.

OLIVEIRA, A.M. 2013. Paleofauna de vertebrados, com ênfase em répteis e mamíferos quaternários da região da Serra da Bodoquena, Mato Grosso do Sul, Brasil. Instituto de Geociências e Ciências Exatas, Universidade Estadual Paulista, Rio Claro, Dissertação de Mestrado, $142 \mathrm{p}$.

OLIVEIRA，A.M.; BECKER-KERBER， B.; CORDEIRO, L.M.; BORGHEZAN, R.; AVILLA, L.S.; PACHECO, M.L.A.F.; SANTOS, C.M.D. 2017. Quaternary mammals from Central Brazil (Serra da Bodoquena, Mato Grosso do Sul) and comments on paleobiogeography and paleoenvironments. Revista Brasileira de Paleontologia, 20(1): 31-44.

OLIVEIRA, E.V.; PEREIRA, J.C. 2009. Intertropical cingulates (Mammalia, Xenarthra) from the Quaternary of Southern Brazil: systematics and paleobiogeographical aspects. Revista Brasileira de Paleontologia, 12(3): 167-178.

OLIVEIRA, E.V.; BARRETO, A.M.F; ALVES, R.M. 2009. Aspectos sistemáticos, paleobiogeográficos e paleoclimáticos dos mamíferos quaternários de Fazenda Nova, PE, nordeste do Brasil. Gaea - Journal of Geoscience, 5(2): 75-85.

OLIVEIRA, E.V.; PORPINO, K.O.; BARRETO, A.F. 2010. On the presence of Glyptotherium in the Late Pleistocene of Northeastern Brazil, and the status of "Glyptodon" and "Chlamydotherium". Paleobiogeographic implications. Neues Jahrbuch Für Geologie Und Paläontologie Abhandlungen, 258(3): 353-363.

OLIVEIRA, L.D.D.; DAMASCENO, J.M. 1987. Registro de novos espécimes de mamíferos fósseis em Lagoa da Cruz - Nova, Cruz, Rio Grande do Norte. In: SBP, CONGRESSO BRASILEIRO DE PALEONTOLOGIA, 10, Rio de Janeiro, Anais, 2: 189-196.

PACHECO, M.L.A.F.; GALANTE, D.; RODRIGUES. F.; LEME, J.M.; BIDOLA, P.;
HAGADORN, W. 2015. Insights into the Skeletozination, Lifestyle, and Affinity of the Unusual Ediacaran Fossil Corumbella. PLoS ONE, 10(3): e0114219.

PAULA COUTO, C. 1973. Edentados fósseis de São Paulo. Anais da Academia de Ciências, 45(2): 261-275.

PAULA COUTO, C. 1978. Mamíferos fósseis do Pleistoceno do Espírito Santo. Anais da Academia Brasileira de Ciências, 50(3): 365-379.

PAULA COUTO, C. 1979. Tratado de Paleomastozoologia. Academia Brasileira de Ciências, Rio de Janeiro, $590 \mathrm{p}$.

PAULO, P.; BERTINI, R. 2013. Registro de Eremotherium Laurillardi (Megatheriidae, Xenarthra) e Stegomastodon Waringi (Gomphotheriidae, Proboscidea) no acervo do Museu de História Natural do Instituto do Trópico Sub-úmido da PUC/ Goiás, Goiânia. Élisée, Revista de Geografia da UEG, 2(1): 63-76.

PÉREZ-CRESPO, V.A.; ARROYO-CABRALES, J.; ALVA-VALDIVIA, L.M.; MORALESPUENTE, P.; IENFUEGOS-ALVARADO, E. 2012. Diet and habitat definitions for Mexican glyptodonts from Cedral (San Luis Potosí, México) based on stable isotope analysis. Geological Magazine, 149(1): 153-157.

PERINI, F.A.; GUEDES, P.G.; NETO, C.R.M.; FRACASSO, M.P.A.; DUHÁ, D.; CAROdOSO, B.; SALLES, L. O. 2009. Carnivores (Mammalia, Carnivora) from the Quaternary of Serra da Bodoquena, Mato Grosso do Sul, Brazil. Arquivos do Museu Nacional, 67(1/2): 119-128.

PITANA, V.G.; ESTEBA, G.I.; RIBEIRO, A.M., CARTELLE, C. 2013. Cranial and dental studies of Glossotherium robustum (Owen, 1842) (Xenarthra: Pilosa: Mylodontidae) from the Pleistocene of southern Brazil. Alcheringa, 37(2): 147-162.

PRADO, J.L.; MENEGAZ, A.N.; SALEMME, M.C. 1987. Los mamíferos de la fauna local Paso Otero (Pleistocene tardio), provencia de Buenos Aires: Aspectos paleoambientales y bioestratigrafícos. Ameghiniana, 24: 217-233.

PRICE, L.I.; CAMPOS, D.A. 1970. Fósseis pleistocênicos no município de Itaboraí, estado do Rio de Janeiro. In: SBG, CONGRESSO 
BRASILEIRO DE GEOLOGIA, 24, Brasília, Anais, 355-358.

PUJOS, F.; SALAS, R. 2004. A systematic reassessment and paleogeographic review of fossil Xenarthra from Peru. Bulletin de l'Institut français d'études andines, 33(2): 331-377.

RINCÓN, A.D.; MCDONALD, G.; SOLÓRZANO, A.; FLORES, M.N.; RUIZ-RAMONI, D. 2015. A new enigmatic Late Miocene mylodontoid sloth from northern South America. Royal Society Open Science, 2: 140256.

ROJAS, O.; MENDONZA, P.L.; MARTÍNEZ, I. 2012. Notas sobre un hallazgo de Megatherium $s p$. (Mammalia, Xenarthra, Megatheriidae) en el curso inferior del río salado (región de antofagasta, Chile). Boletín del Museo Nacional de Historia Natural, 61: 75-84.

SAINT-ANDRE, P.; DE IULIIS, G. 2001. The smallest and most ancient representative of the genus Megatherium Cuvier, 1796 (Xenarthra, Tardigrada, Megatheriidae), from the Pliocene of the Bolivian Altiplano. Geodiversitas Publications Scientifiques du Muséum national d'Histoire naturelle, 23(4): 625-645.

SALLES, L.O.; CARTELLE, C.; GUEDES, P.G.; BOGGIANI, P.C.; JANOO, A.; RUSSO, C.A.M. 2006. Quaternary mammals from Serra da Bodoquena, Mato Grosso do Sul, Brazil. Rio de Janeiro: Museu Nacional, 12 p. (Boletim, 541).

SCHEFFLER, S.M.; MARTINS, G.R.; KASHIMOTO, E.M.; OLIVEIRA, A.M. 2010. Revisão sobre a paleontologia no estado do Mato Grosso do Sul: fósseis e afloramentos descritos. Brazilian Geographical Journal, 1: 65-99.
SCILLATO-YANÉ, G.J.; CARLINI, A.A.; TONNI, E.P.; NORIEGA, J.I. 2005. Paleobiogeography of the late Pleistocene pampatheres of South America. Journal of South American Earth Sciences, 20: 131-138

SILVA, F.M.; FILGUEIRAS, C.F.C.; BARRETO, A.M.F.; OLIVEIRA, E.V. 2010. Mamíferos do Pleistoceno Superior de Afrânio, Pernambuco, nordeste do Brasil. Quaternary and Environmental Geosciences, 2(2): 1-11.

SIMPSON, G.G.; PAULA COUTO, C. 1981. Fossil mammals from the Cenozoic of Acre, Brazil. III. Pleistocene Edentata Pilosa, Proboscidea, Sirenia, Perissodactyla and Artiodactyla. Iheringia, Série Geológica, 6: 11-73.

VIZCAÍNO, S.F.; DE IULIIS, G.; BARGO, M.S. 1998. Skull Shape, Masticatory Apparatus, and Diet of Vassallia and Holmesina (Mammalia: Xenarthra: Pampatheriidae): When Anatomy Constrains Destiny. Journal of Mammalian Evolution, 5(4): 291-322.

ZURITA, A.E.; OLIVEIRA, E.V.; TORIÑO, P.; RODRIGUEZ-BUALÓ, S.M.; SCILLATO-YANÉ， G.J.; LUNA， C.; KRAPOVICKAS, J. 2011. On the taxonomic status of some Glyptodontidae (Mammalia, Xenarthra, Cingulata) from the Pleistocene of South America. Annales de Paléontologie, 97:63-83.

ZURITA, A.E.; MIÑO-BOILINI, Á.R.; FRANCIA, A.; ARENAS-MOSQUERA, J.E. 2012. The Pleistocene Glyptodontidae Gray, 1869 (Xenarthra: Cingulata) of Colombia and some considerations about the South American Glyptodontinae. Revista Brasileira de Paleontologia, 15(3): 273-280.

\section{Endereço dos autores:}

Thaís Rabito Pansani - Departamento de Biologia, Universidade Federal de São Carlos, campus Sorocaba, Rodovia João Leme dos Santos (SP-264), Km 110, s/n - Itinga, CEP 18052-780, Sorocaba, SP, Brasil. Bolsista voluntária PIBIC-UFSCar.E-mail: thais-pansani@hotmail.com

Alessandro Marques de Oliveira - Universidade Federal do ABC, Av. dos Estados, 5001, Bangu, CEP 09210-580, Santo André, SP, Brasil.E-mail: biolessandro@gmail.com

Mírian Liza Alves Forancelli Pacheco - Departamento de Biologia, Universidade Federal de São Carlos, campus Sorocaba, Rodovia João Leme dos Santos, (SP-264), Km 110, s/n - Itinga, CEP 18052-780, Sorocaba, SP, Brasil.E-mail: forancelli.ufscar@gmail.com

Artigo submetido em 1 de agosto de 2016, aceito em 29 de março de 2017. 